\title{
Keep on Rockin' in a (Plastic-)Free World: Collective Efficacy and Pro-Environmental Intentions as a Function of Task Difficulty
}

\author{
Gerhard Reese ${ }^{1, *}$ and Eva A. Junge ${ }^{2}$ \\ 1 Environmental Psychology Unit, University of Koblenz-Landau, Fortstr. 7, 76829 Landau, Germany \\ 2 Centre for Sustainability Studies, Lund University, SE 22100 Lund, Sweden; evajunge@posteo.de \\ * Correspondence: reese@uni-landau.de; Tel.: +49-6341-2803-1460
}

Academic Editors: Gerrit Antonides and Marc A. Rosen

Received: 27 October 2016; Accepted: 24 January 2017; Published: 1 February 2017

\begin{abstract}
Collective efficacy-the belief that one's group is capable of affecting relevant aspects of its environment-has been highlighted as an important predictor of sustainable behavior. It increases people's collective action tendencies, and is important for fostering environmental behavioral change beyond self-efficacy beliefs. The current study addresses two primary goals. First, we tested whether the difficulty of a task increased collective efficacy, and thereby environmental intentions. Second, we explored how collective and self-efficacy in concert predict such intentions. In a combined field-and-survey study, 165 voluntary participants took part in a plastic reduction challenge that was pretested as easy, moderate, or difficult. After being confronted with the task, participants completed an online questionnaire in which, among other variables, specific and general self-efficacy, collective efficacy, and pro-environmental intentions were measured-both general and plastic-reduction specific. Results revealed that (a) collective efficacy was significantly stronger when task difficulty was moderate rather than easy or difficult; and (b) that through specific collective and self-efficacy perceptions, sustainable intentions were gauged - even when controlling for attitudes and social norms. These findings suggest that collective efficacy beliefs are particularly relevant for attaining environmental goals that are neither too easy nor too difficult, and could thus be valuable for communication and policy strategies.
\end{abstract}

Keywords: collective efficacy; self-efficacy; sustainable behavior; pro-environmental behavior; plastic use; social norms

\section{Introduction}

In the face of climatic change and increasingly severe environmental problems, it is evident that humanity needs sustainable solutions to respond to such global challenges. Besides policy measures and transnational cooperative efforts of various stakeholders (e.g., nations in the frame of global climate summits, e.g., [1,2]), one part of the solution is individuals and their compliance with sustainable behavioral options [3]. In fact, various approaches in environmental psychology suggest pathways towards and barriers of pro-environmental action (e.g., [4-6]), and provide compelling evidence for individual psychological processes determining pro-environmental responses. However, it is also apparent that individual contributions, such as avoiding the use of plastic bags during grocery shopping, are limited in their potential to effect change, if they are not carried out by many. Therefore, identifying predictors of collective behavior represents an important endeavor to understand and motivate pro-environmental action. One of these predictors is collective efficacy-the belief that one's group is capable of affecting important aspects of its environment (in the broader sense of the term, not limited to the natural environment; [7]). The current study builds up on 
this idea that collective efficacy is an important predictor of pro-environmental action ([8-10]). Yet, we extend previous research on the link between collective efficacy and pro-environmental action, testing conditions under which environmental collective efficacy would be increased, and be most predictive of pro-environmental intentions. Specifically, we argue that task difficulty-i.e., the difficulty to perform a specific pro-environmental behavior-affects environmental collective efficacy beliefs, and thereby, pro-environmental intentions. In addition, we draw from recent research that suggests that environmental collective efficacy beliefs raise environmental self-efficacy beliefs, which ultimately predict sustainable behavioral options [11,12]. In the context of plastic-reduction behavior, we developed a combined field-survey-experiment to test these assumptions.

\subsection{The Plastic Issue}

Production and usage of plastic have been continuously rising since its discovery, reaching a yearly total of about 220 million tons worldwide (of which about a quarter is being consumed in Europe). For its production, high amounts of non-renewable, extremely environmentally-damaging resources such as petroleum, ethylene, and coal are needed [13]. Not only does the extraction-process of these resources yield very harmful consequences, but because of plastic's incapability to decompose, it remains in the environment for up to 500 years (see for example [14]). A huge amount of this gathers in the oceans, where it has an uncountable amount of severe impacts on the environment and its flora and fauna. Apart from the detrimental impacts of plastic(-waste) on the environment and its pollution of rural as well as urban areas, plastic has even been detected in humans and is suspected of causing various health risks [15]. Yet, despite our awareness about the various undesirable consequences plastic already has today, the quantity of plastics produced worldwide in the first decade of this century is equivalent to the total world production in the previous century [16]. Therefore, governments of various states (e.g., Ireland) put a stop on the waste of plastic bags in stores by introducing a fee for each bag, or even completely banning them. While such regulations are a valuable contribution to the effort of global reduction of plastic waste, they force individuals into changing their behavior. Beyond such regulatory approaches, Cherrier [17] has found that voluntary anti-consumption involves environmental consciousness, and that repeatedly taking one's own shopping bag leads to greater reflection on the environmental impact of plastic bag consumption. This underlines the need for tools that facilitate behavioral change complementing political top-down approaches that may evoke reactance.

\subsection{Collective Efficacy and Pro-Environmental Action}

Human behavior is significantly determined by individuals' belief in their self-efficacy. This personal sense of their own capability to produce and regulate events in their lives is a major incentive to act $[18,19]$. In fact, a lack of perceived self-efficacy can prevent individuals from becoming active [20], and people even tend to avoid those activities they believe exceed their coping capabilities [21]. In turn, a stronger belief in one's self-efficacy relates to stronger efforts and resilience to adversity [22], better job performance [23], higher satisfaction with relationships [24], and better athletic performance [25]. With such broad effects of self-efficacy on human behavior (but see [25], for a critical analysis of self-efficacy effects), it is plausible and empirically supported that self-efficacy significantly predicts pro-environmental behavior. In fact, the more individuals perceive themselves as self-efficacious, the more prone they are to act in favor of the natural environment $[4,12,26]$. Specifically, recent evidence shows that self-efficacy beliefs can explain spill-over effects from easy sustainable actions to more difficult ones [26]. Lauren, Fielding, Smith, and Louis [26] showed that previous engagement in a relatively easy pro-environmental task (e.g., turning off water while brushing teeth) was related to increased self-efficacy, which in turn predicted stronger engagement in more difficult tasks nine months later (e.g., actual installment of water-saving devices). In other words: Increasing individuals' belief in their efficacy through acting pro-environmentally could foster future pro-environmental action, even in other contexts. 
Yet, it is important to acknowledge that efficacy beliefs are not limited to individuals' personal self, but also connected to their collective self. Individuals can perceive their group as collectively efficacious [19]. The belief that one's own group is capable of affecting important aspects of its environment is particularly relevant for understanding collective action, such as responses to environmental degradation. Evidently, no individual alone will be able to mitigate climate change or combat biodiversity loss. Consequently, it is necessary to understand the social processes that enable individuals to act in concert (see also [27]). Thus, being a member of a group can change our beliefs about which goals we can achieve. While as an individual, using one plastic bag more or less cannot solve the plastic issue, people may feel that as a group, they have the power to make a difference.

Recent studies suggest that this perceived environmental collective efficacy is particularly predictive of pro-environmental action. For example, Barth and colleagues [8] analyzed intentions to use electric vehicles both in a purchasing and sharing scenario. Among other predictors such as descriptive and ingroup norms, personal cost-benefit analyses, knowledge and experience, collective efficacy was assessed and predicted stronger intentions both in the purchasing and the sharing scenario. Similarly, collective efficacy beliefs were more strongly connected to the choice of a more environmentally sustainable travel option than self-efficacy beliefs [10]. These authors also showed environmental collective efficacy beliefs to be a stronger predictor of peoples' willingness to pay for environmental protection than self-efficacy and attitudes [9]. Morton and colleagues [28] assessed collective efficacy as individual's perception of their group being efficacious in mitigating or dealing with the consequences of climate change. Here, collective efficacy was a significant predictor of private-sphere environmental actions (i.e., reducing household waste and non-green energy consumption). Earlier, Homburg and Stolberg [29] found that appraisals of collective efficacy, rather than self-efficacy, predicted pro-environmental intentions (see also [30]). While this evidence suggests that we should focus on collective efficacy in order to promote sustainable behavior, more recent evidence by Jugert and colleagues [12] suggests that a better understanding of the mechanisms linking efficacy perceptions and sustainable behavior is needed. Specifically, these authors argued that collective efficacy exerts its effects on sustainable behavior through raising perceptions of self-efficacy. This process is explained by the model of group-based control that postulates individuals can derive personal benefits (e.g., self-efficacy beliefs) from social groups because groups can make them feel personally capable and in control [31,32]). In fact, Jugert et al. [12] could show that through collective efficacy, individuals came to feel in control of their outcomes: People's intention to act was enhanced through providing a sense of efficacy transferred from the group to the self. Similarly, using a qualitative research approach, Cocking and Drury [11] found that collective efficacy led to a feeling of personal empowerment. Thus, with collective and self-efficacy being strong and closely intertwined predictors of pro-environmental action, the current research seeks to understand under which conditions their effect on sustainable behavior is particularly prominent.

\subsection{Task Difficulty and Challenge Framing}

Under which conditions do people feel most collectively efficacious to act pro-environmentally? To our knowledge, there is little research specifically addressing this question. However, given that collective efficacy is an important predictor for achieving societal change [7], answering this question could provide insights into a powerful mechanism of sustainable action. Based on the idea that task difficulty affects individuals' performance following an inverted U-shape ([33]; for more recent accounts, see [34,35]), we deem it plausible to assume that similarly, collective and self-efficacy beliefs that precede performance may depend on the difficulty of a task at hand. According to research on the inverted U-model, a moderate level of difficulty would result in strongest motivation to act compared with a task that is too simple or too difficult (cf. [36]). Similarly, we predict that individuals would perceive efficacy particularly for those tasks that are neither too difficult nor too easy. First, people may either expect that fewer others would engage in the behavior when it is too difficult. Supporting this assumption, Van Zomeren et al. [37] found that social action support significantly predicted collective 
efficacy. Second, behaviors that are easy usually have a weaker environmental impact per se. It is likely that people believe actions that are too easy (e.g., refraining from plastic bags) to be unlikely to make a big difference in environmental issues, even if they are collectively practiced. In other words, when actions are too easy, the (potential) success may not translate into feeling collectively efficacious. In short, this suggests that efficacy beliefs would be strongest for medium difficulty tasks.

\subsection{Present Research}

The present research thus provides two novel contributions to sustainable behavior: First and foremost, we test whether task difficulty affects efficacy beliefs, and their relation to pro-environmental intentions. Drawing from our reasoning above, we first hypothesize that (a) efficacy-both self-and collective-is highest for tasks perceived to be of medium, rather than low or high, difficulty. In a second step, we (b) hypothesize that collective efficacy is particularly predictive of pro-environmental intentions through raising perceptions of self-efficacy. In statistical terms, we thus expect a serial mediation effect such that medium task difficulty increases collective efficacy, which in turn predicts higher self-efficacy. Ultimately, the higher perceived self-efficacy, the stronger individuals' sustainability intentions. Finally, we test our predictions both with plastic-reduction-specific beliefs and intentions as well as with more general sustainability beliefs and intentions.

The second contribution is our methodological approach. We combine a field experimentmanipulating task difficulty in a playful approach through widely distributed cards presenting "plastic reduction challenges" - with an online survey that assesses our dependent (collective efficacy, pro-environmental intentions) and control variables (see below). This methodology is insofar innovative as it maximizes external validity of the independent variable task difficulty while providing internal validity of our primary dependent variables. Finally, in order to test the unique contribution of our proposed model, we assessed additional constructs that relate to pro-environmental action. Descriptive and injunctive norms (e.g., [38-42]) as well as attitudes (e.g., [5]) were selected for this study.

\section{Materials and Methods}

\subsection{Design and Pretest of the Plastic Challenge Cards}

In order to vary task difficulty, we designed playing cards with different challenges. The basic idea was that people receiving the playing cards were challenged to behave in a more sustainable way and after successful completion, sign the card and hand it on to challenge another person. The cards were dark green and had the size of usual playing cards, so that they would easily fit into any purse or pocket. The challenges were printed on the top of the cards, followed by empty fields for participants' signatures. Participants were prompted to sign only once they completed the specific challenge that was displayed on the card. Depending on the degree of challenge difficulty, the following wording was used: "I carried my groceries home in either a fabric bag, a backpack, or a basket" (easy), "While shopping, I bought all fruits and vegetables without plastic wrapping, and I consequently brought them home in either a fabric bag, a backpack, or a basket." (medium), and "I did my entire shopping entirely without plastic. This means, that I neither bought plastic bags of any sorts nor did I buy any other sort of plastic wrapping" (hard). On the flipside of the card, some information about plastic use and its environmental problems was displayed. This information was identical across the conditions. Figure 1 displays the medium condition of the task difficulty (We also manipulated the frame of the wording with the goal of increasing collective efficacy [individual vs. collective wording]. However, this variation had no effect [neither main nor interaction with task difficulty] on any of our dependent variables and is thus not reported on further).

We conducted a pretest to make sure that the challenges we crafted were indeed selective so that participants' evaluation coincided with our proposed task difficulty. A brief questionnaire including the challenges and the question "How difficult do you believe the following challenge is?", rated from 1 (very easy) to 7 (very difficult) was distributed to $N=60$ passers-by in a public pedestrian 
area in a German town. In line with our expectations, we found a significant and strong main effect of task difficulty, $F(2,57)=86.41, p<0.001, \eta^{2} p=0.75$, such that the easy task was rated easiest $(M=1.56, S D=1.12)$, the difficult task as most difficult $(M=5.02, S D=1.7)$, and the moderate task falling in between $(M=2.54, S D=1.54)$. Thus, the pretest shows that the challenges were perceived as differently difficult.

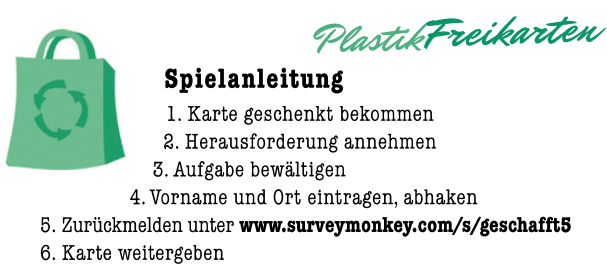

Warum weniger Plastik?

Wenn du und alle anderen Menschen in
Deutschland Mehrwegtaschen verwenden,
können wir pro Jahr über 185.000 Tonnen
Kunststoffmüll verhindern.
Durch die Vermeidung von Plastik können
wir gesundheitlichen Beeinträchtigungen
wie bspw. Herzkreislauferkrankungen, Le-
berproblemen, Diabetes und Unfruchtbar-
keit durch hormonelle Störungen vorbeugen.
Im Nordpazifik treibt seit Jahrzehnten ein
Müllstrudel, der mittlerweile so groß ist wie
Zentraleuropa. Durch die Nutzung von
Mehrwegtaschen können wir unseren Teil
zur Rettung unserer Meere beitragen.

(a)

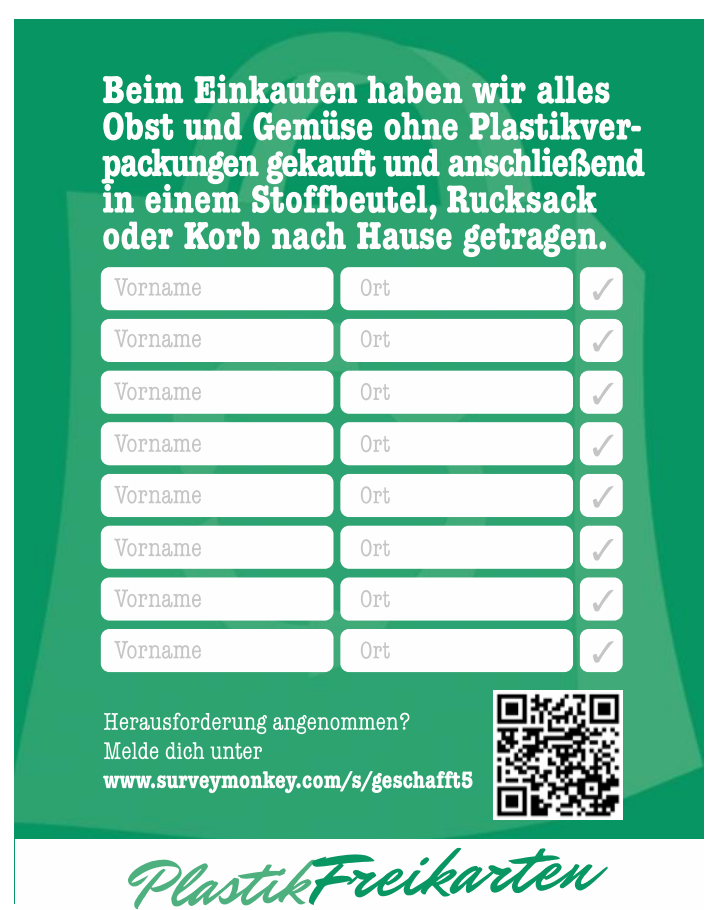

(b)

Figure 1. Back (Panel a) and front (Panel b) of the "medium difficulty" plastic challenge card. Translation left panel, upper text: "Instructions: 1. Receive card, 2. Accept challenge, 3. Conduct challenge, 4. Enter first name and place, check, 5. Go to website, 6. Hand over card"; Left panel, boxes: "Why less plastic?"; Box 1: "If you and all other people in Germany would use reusable bags, more than 185.000 tons of plastic waste could be prevented", Box 2: "Through reducing plastic, health problems such as coronary diseases, liver problems, diabetes and infertility through hormone dysregulation could be prevented"; Box 3: "In the northern pacific, a waste vortex the size of central Europe has developed over the last decades. By using reusable bags, we can contribute to save our seas"; Right panel, main text: "For our shopping, we bought all our fruits and vegetable without plastic wrapping and consequently brought them home in either a fabric bag, a backpack or a basket"; Right panel, fields: "First name, place"; Right panel, lower text: "Challenge accepted? Go to www.surveymonkey.com/s/geschafft5".

\subsection{Research Participants and Procedure}

The plastic challenge cards were distributed in various towns and cities across Germany. Altogether, 6000 cards were given out to people directly in face-to-face interactions in May and June 2015. 165 participants (93 female, 58 male, 14 did not indicate gender, $M_{\text {age }}=31.7, S D=12.4$ ) participated in the online survey after completion of the challenge. Of these, 73 (44\%) indicated to be students. If not indicated otherwise, all items in the online questionnaire were administered using 7-point Likert scales reaching from 1 (not true at all) to 7 (completely true) unless otherwise stated, and for each construct, item scores were combined into a single mean score. 


\subsection{Material}

- Collective efficacy. Collective efficacy was measured with two general items "I am optimistic that we as plastic challenge participants can protect the environment together"; "We as plastic challenge participants have the capability to protect the environment"; $(r=0.90)$ and one plastic-specific item ("I think we as plastic challenge participants can collectively protect the environment with reducing plastic usage"). The items were formulated similarly to previous items assessing efficacy beliefs $[11,27]$ to fit the current study design.

- Self-efficacy. We gauged self-efficacy with two general items ("I am optimistic, that I can protect the environment"; "I am capable of protecting the environment"; $r=0.87$ ) and one plastic-specific item ("I think that I am capable of protecting the environment by means of my personal plastic reduction"). These were adapted questions from a questionnaire by [5] (In line with other researchers, we measured efficacy perceptions with regard to individuals' confidence in their ability (e.g., [7,8,29]) rather than action-/behavior-directed, as Bandura's conceptualization would suggest. Hanss and colleagues ([43]) provide a comprehensive discussion of different approaches to measure and conceptualize efficacy).

- Behavioral intentions. We tapped behavioral intentions with three general items ("I will try to reduce my eco footprint in the next month"; "I intend to behave more environmentally friendly in the following month"; and "I am planning to waste less of natural resources in the next month"; $\alpha=0.95$ ) and one plastic-specific item ("I intend to abstain from plastic while shopping"). These items were taken from the Green Behavior Intention Scale by [44].

- Descriptive norms. We inquired about descriptive norms with four items, adapted from [40]. Participants were asked to estimate the frequency with which "inhabitants of Germany try to reduce plastic while shopping"; "inhabitants of their city try to reduce plastic while shopping"; "friends and family try to reduce plastic while shopping"; and "the person that challenged them tries to reduce plastic while shopping". $(\alpha=0.79)$.

- Injunctive norms. We measured injunctive norms with four items: "If I reduced plastic while shopping, people that matter to me would (disapprove-approve)"; "Most people that matter to me think that consuming less plastic while shopping is (undesirable-desirable)"; "Most people that matter to me think that I should (not at all-very much) advocate for plastic consumption reduction while shopping"; and "The person that challenged me to this card considers plastic reduction while shopping as (irrelevant-very relevant)" $(\alpha=0.66)$.

- Attitudes. In order to measure attitudes towards plastic use, we provided seven bi-polar items on which participants were asked to rate how they perceive plastic consumption while shopping ("good-bad", "foolish-wise", "harmful-useful", uncomfortable-comfortable", "dissatisfactory—satisfactory", "disadvantageous—advantageous", and "negative-positive"). Those items were adapted from [45] $(\alpha=0.79)$.

- Demographics. At the end of the questionnaire, we gauged participants' age, gender, socio-economic status, and education status (student vs. non-student).

\section{Results}

Means, standard deviations, reliabilities, and bivariate correlations for all measures across conditions are reported in Table 1. Number of participants by condition is depicted in Table 2. As can be seen, the highest number of participants indicated having completed an easy challenge, the lowest number of participants having completed the difficult challenge, and a number of participants in-between completed the moderate challenge. Regarding our hypotheses, we first tested the effect of task difficulty on collective and self-efficacy beliefs, both for plastic-specific and general items. A MANOVA comparing the means of efficacy revealed significant main effects for plastic-specific $\left(F[2,156]=3.26, p=0.041, \eta^{2} p=0.04\right)$ and general $\left(F[2,156]=3.82, p=0.024, \eta^{2} p=0.05\right)$ collective 
efficacy. For self-efficacy, there was a tendency for a main effect for plastic-specific $(F[2,156]=2.61$, $\left.p=0.077, \eta^{2} \mathrm{p}=0.03\right)$ self-efficacy, but no effect for general self-efficacy $(F<1)$.

As can be seen in Table 2, and in line with our predictions, collective efficacy beliefs were most strongly pronounced when the task difficulty of the plastic reduction challenge was moderate. Comparing the moderate condition with both the easy and the difficult condition revealed a significant main effect for both plastic-specific $\left(F[1,158]=6.5, p=0.012, \eta^{2} \mathrm{p}=.04\right)$ as well as general collective efficacy $\left(F[1,158]=7.65, p=0.006, \eta^{2} \mathrm{p}=0.05\right)$, such that in the moderate condition $($ Mplastic $=5.14$, SDplastic $=1.42 ;$ Mgeneral $=5.09$, SDgeneral $=1.48)$, collective efficacy was significantly higher than in both other conditions (Mplastic $=4.44$, SD plastic $=1.77 ;$ Mgeneral $=4.34$, SDgeneral $=1.72$ ). For self-efficacy, however, there was a tendency for plastic-specific efficacy $(F[1,158]=3.81, p=0.053$, $\eta^{2} \mathrm{p}=0.02$ ) such that in the moderate condition (Mplastic $=6.14$, SDplastic $=0.97$ ), plastic-specific efficacy was descriptively higher than in both other conditions (Mplastic $=5.68$, SDplastic $=1.63$ ). There was no effect for general self-efficacy, $F<1$. These findings suggest that the difficulty of the plastic reduction task affected collective efficacy, but less so self-efficacy.

Table 1. Means, standard deviations, and bivariate correlations for all dependent and control variables, across experimental groups.

\begin{tabular}{|c|c|c|c|c|c|c|c|c|c|c|c|}
\hline & $M$ & $S D$ & 1. & 2. & 3. & 4. & 5. & 6. & 7. & 8. & 9. \\
\hline $\begin{array}{l}\text { 1. Collective } \\
\text { efficacy-plastic }\end{array}$ & 4.69 & 1.69 & - & $0.91 *$ & $0.51 *$ & $0.53 *$ & $0.40 *$ & $0.38 *$ & $0.31^{*}$ & $0.23 *$ & 0.27 * \\
\hline $\begin{array}{l}\text { 2. Collective } \\
\text { efficacy—general }\end{array}$ & 4.61 & 1.67 & & - & $0.43^{*}$ & $0.58^{*}$ & $0.33^{*}$ & $0.40^{*}$ & $0.31^{*}$ & $0.16^{*}$ & $0.25 *$ \\
\hline 3. Self-efficacy一plastic & 5.84 & 1.44 & & & - & $0.60 *$ & $0.48^{*}$ & $0.24^{*}$ & $0.50^{*}$ & 0.13 & $0.19 *$ \\
\hline 4. Self-efficacy-general & 5.36 & 1.51 & & & & - & $0.33 *$ & $0.32 *$ & $0.45^{*}$ & $0.08^{*}$ & $0.09 *$ \\
\hline $\begin{array}{l}\text { 5. Sustainable } \\
\text { intentions-plastic }\end{array}$ & 6.03 & 1.21 & & & & & - & $0.65^{*}$ & $0.51^{*}$ & $0.28 *$ & $0.34^{*}$ \\
\hline $\begin{array}{l}\text { 6. Sustainable } \\
\text { intentions-general }\end{array}$ & 5.42 & 1.3 & & & & & & - & $0.33^{*}$ & $0.23 *$ & $0.33^{*}$ \\
\hline 7. Attitudes & 6.15 & 0.78 & & & & & & & - & $0.23 *$ & 0.29 * \\
\hline 8. Descriptive norms & 3.91 & 0.99 & & & & & & & & - & 0.37 * \\
\hline 9. Injunctive norms & 5.98 & 0.72 & & & & & & & & & - \\
\hline
\end{tabular}

Table 2. Means and standard deviations for collective and self-efficacy as a function of task difficulty.

\begin{tabular}{cccc}
\hline \multirow{2}{*}{ Measure } & \multicolumn{3}{c}{ Task Difficulty } \\
\cline { 2 - 4 } & Easy & Medium & Difficulty \\
\cline { 2 - 4 } & $\boldsymbol{M ( S D )}$ & $\boldsymbol{M}(S D)$ & $\boldsymbol{M}(S D)$ \\
\hline Collective efficacy-plastic & $4.40(1.76)$ & $5.14(1.42)$ & $4.58(1.84)$ \\
Collective efficacy-general & $4.31(1.72)$ & $5.1(1.48)$ & $4.46(1.75)$ \\
Self-efficacy-plastic & $5.77(1.55)$ & $6.14(0.97)$ & $5.42(1.86)$ \\
Self-efficacy-general & $5.29(1.6)$ & $5.46(1.26)$ & $5.33(1.77)$ \\
$N$ (participants) & 78 & 58 & 29 \\
\hline
\end{tabular}

Note: A higher score indicates higher efficacy, except for last line. Chi ${ }^{2}$-Analysis shows that cases are not equally distributed between levels of task difficulty, $X^{2}(2,165)=22.07, p<0.001$.

In a second step, we tested our serial mediation hypothesis. Specifically, we predicted an indirect effect of task difficulty on sustainable intentions via collective and self-efficacy. To test this assumption, we ran two statistical models, one each for plastic-specific and general efficacy beliefs and sustainable intentions. We conducted analyses of the assumed indirect effects with bootstrapping resamples, computing confidence intervals of an indirect effect-a method preferred to the normal theory approach, which assumes the often violated assumption of normality of the sampling distribution of 
the indirect effect [46]. An indirect effect as the one we propose here is deemed significant with $95 \%$ confidence if " 0 " is not within the $95 \%$ confidence interval. To test our indirect effects, we used the SPSS process macro (Model 6; [46]) with 5000 bootstrapping resamples. Task difficulty was recoded so that the moderate condition could be compared with both the easy and the medium conditions that were collapsed into one group $(1=$ moderate task difficulty, $0=$ otherwise $)$ before analyses.

In line with our expectations, there was a significant sequential indirect effect of task difficulty via plastic-specific collective and self-efficacy on plastic reduction intentions, $B=0.10, S E=0.06$, bias-corrected $95 \% C I[0.02 ; 0.24]$. This effect remained significant even after controlling for covariates (injunctive and descriptive norms, attitudes), $B=0.04, S E=0.03$, bias-corrected 95\% $C I[0.003 ; 0.11]$. Thus, a moderate task difficulty-compared with an easy or difficult task-increased sustainable intentions through a process of increased collective efficacy and subsequently increased self-efficacy (see Figure 2). To gauge additional evidence of this proposed process, we also tested the reverse pathway that task difficulty first increased self-efficacy, which in turn increased collective efficacy to predict sustainable intentions. This model, however, was substantially weaker, $B=0.04, S E=0.03$, bias-corrected $95 \%$ CI $[0.005 ; 124]$, and non-significant when controlling for the covariates above, $B=0.01, S E=0.01$, bias-corrected $95 \% C I[-0.003 ; 0.06]$, supporting our suggested sequence.

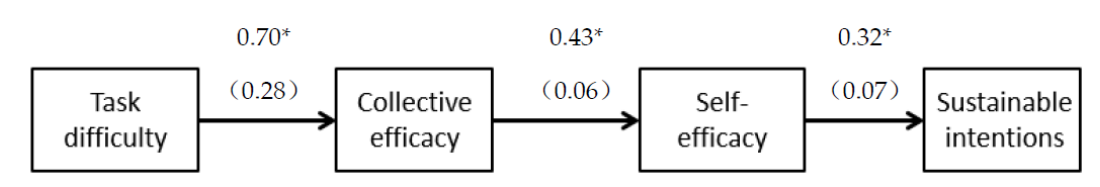

Figure 2. Indirect effect of task difficulty via collective and self-efficacy for plastic-specific efficacy and intentions. Note: $N=155$, due to missing values. Coefficients are unstandardized, with standard errors in brackets. Model without controlling for the covariates.

We also tested the model for general collective and self-efficacy on general sustainable intentions. For these, there was no significant sequential indirect effect, $B=0.05, S E=0.04$, bias-corrected $95 \% C I$ $[-0.001 ; 0.15]$. Controlling for the covariates did not improve the indirect effect, $B=0.02, S E=0.03$, bias-corrected $95 \% C I[-0.02 ; 0.10]$. Also, there was no reverse mediation with self-efficacy first, followed by collective efficacy, $B=0.03, S E=0.04$, bias-corrected $95 \% C I[-0.04 ; 0.13]$. Note, however, that there was an indirect effect of task difficulty via collective efficacy alone, $B=0.19, S E=0.09$, bias-corrected $95 \%$ CI [0.06; 0.42], suggesting that collective efficacy may indeed play a more important role for such collective tasks than self-efficacy.

The separate steps of the serial mediation were also significant: There was an indirect effect of task difficulty on self-efficacy via collective efficacy for plastic specific efficacy, $B=0.15, S E=0.06$, bias-corrected $95 \% C I[0.04 ; 0.30]$, as well as for general sustainable efficacy, $B=0.20, S E=0.08$, bias-corrected $95 \%$ CI [0.06; 0.37].

For intentions, there was an indirect effect of collective efficacy on plastic specific intentions via self-efficacy, $B=0.14, S E=0.05$, bias-corrected $95 \% C I[0.06 ; 0.25]$, but not for general sustainable intentions, $B=0.06, S E=0.04$, bias-corrected $95 \% C I[-0.02 ; 0.15]$, resembling the non-significant full sequential model above.

\section{Discussion}

Responding to climate and environmental crises requires the collective efforts of many. It is therefore necessary to understand the conditions under which people perceive a collective to be effective in reaching its goal $[7,19]$. The current study contributes significantly to this understanding, showing that collective efficacy beliefs depend on the perceived difficulty of a particular task. When the task difficulty was medium, rather than low or high, collective efficacy beliefs were strongest, and in turn, most predictive of pro-environmental intentions. In addition, this experiment suggests that collective efficacy beliefs can influence self-efficacy beliefs that in turn result in sustainable behavior 
(see also [12]). Thus, even without a direct effect of the task difficulty manipulation on sustainability, it did indirectly make a difference via increasing participants' collective and self-efficacy perceptions. This evidence is both theoretically as well as practically relevant.

First, the current study suggests that collective efficacy is not always such a powerful predictor as suggested in previous research. Apparently, a task that is perceived to be of moderate difficulty seems appropriate to induce a maximum of collective efficacy beliefs. Our findings thus parallel previous research on the effects of task difficulty on performance (e.g., [34-36]), suggesting that task-related changes in performance may be due to changes in collective efficacy beliefs. This is relevant for research on collective and pro-environmental action as it shows that depending on how difficult societal challenges are, different feelings of what people can achieve emerge. Thus, this study may provide a starting point for more rigorously assessing the conditions that make people trust (or not trust) in their capabilities to foster change, and thus has clear implications for sustainability policy making (see below).

Second, the sequential model we propose here suggests that effects of collective efficacy should be understood as depending on changes in self-efficacy. As Jugert et al. [12] suggest, self-efficacy seems a necessary ingredient that drives the effects of collective efficacy on behavioral outcomes. Importantly, in our experiment, this sequential indirect effect was only statistically significant for plastic-specific behaviors-not surprisingly, given that the challenge we asked participants to perform dealt with the reduction of plastic use. However, for research on spillover effects (for a review, see [47]), it is helpful to know that task difficulty of a topic-specific challenge (as in our case, a plastic reduction task) can also increase general collective efficacy beliefs that might in turn enhance pro-environmental behavior in other domains-a finding in line with [26], who show that self-efficacy beliefs mediate between less difficult and more difficult behaviors. Our findings nicely complement this research, suggesting that such spill-over can also be mediated via collective efficacy beliefs.

Interestingly, while our model received support such that strongest efficacy was reported for a medium task, most participants who took part in the survey completed the easy task. This is not surprising, given that this task, compared with the medium and difficult task, was the least time consuming task. Participants could just do this casually, without extended planning of grocery shopping. In other words, an easy task may result in stronger response rates but lower efficacy beliefs and a moderate task in lower response rates but higher efficacy beliefs-that in turn predict future intentions and behavior. This information may be particularly relevant to policy makers and practitioners working in sustainability campaigning.

- Policy implications. The trade-off between responses to more or less difficult tasks and perceived (collective) efficacy is informative for policy making as it shows that people may more easily engage in behaviors that cost little but feel more efficacious through performing somewhat more difficult behaviors. The latter, however, may be performed by fewer individuals. Thus, it is important to identify sustainable actions that are moderately difficult but at the same time appealing enough on other dimensions (e.g., time efficient) in order to result in stronger response rates. We thus believe that the current experiment suggests applications in the field of policy communication and campaigning. Based on the current findings, it seems helpful to craft policy measures - or at least their way of communication-in a way that enables individuals to collectively address issues through moderate "behavioral challenges". For example, providing a community with information about moderate behaviors needed to achieve energy autonomy could increase their collective efficacy to actually engage in such an endeavor, increasing people's self-efficacy to ultimately act. Focus groups or representative surveys could help in identifying broad concepts of difficulty in the public. Of course, such policy measures need to be flanked by additional psychological concepts such as subjects of justice [48], intergenerational and ecological justice appraisals [49], as well as others. Yet, gaining knowledge about possibilities to directly encourage individuals through raising their efficacy beliefs represent an encouraging path to take. Specifically, it could be a fruitful step to investigate the underlying dimensions of difficulty 
(e.g., economic cost, psychological cost, time, effort, etc.) to understand the difficulty-efficacy trade-off described above.

- Limitations. The limitations of this work also deserve comment. It is in the nature of field experiments that we cannot control every potential confounding variable. First of all, cards were handed out to participants in public spaces with the plea to fill out the online survey (link and QR-Code given at the bottom of the cards) after completing the challenge. Consequently, there was no direct means of ensuring that participants in the different conditions participated to equal extents. This implies that we do not know about the individual reasons of why participants did or did not fill out the survey. Additionally, we do not know how much time passed between participants' receiving their card and engaging in the challenge neither do we know the time lag between completion of the task and survey participation. These points notwithstanding, it is even more intriguing that the difficulty of the task influenced beliefs and intentions in the subsequent online questionnaire. A second issue that needs clarification is the perceived difficulty of the task. While our pretest was successful in showing that our manipulation resulted in the expected difficulty ratings (yet, with the medium difficulty being statistically somewhat closer to the easy than the difficult task), future studies should implement a methodology that allows participants to select their preferred task difficulty. In our study, participants were confronted with one of the three difficulties at random so that it is likely that "moderate difficulty" could be easy for some, and hard for others. In this line, it is important to mention that the different tasks yielded differing environmental impacts depending on consumption patterns and specific products that participants would purchase while completing the challenge (e.g., glass containers might, under certain circumstances, yield worse environmental impact than the equivalent plastic container). We assume that the great majority of participants did not possess the necessary background knowledge to take this into consideration when consequently rating one's own efficacy beliefs concerning the behavior change. It is also possible that people from various socio-structural backgrounds (e.g., students vs. non-students) perceive task difficulty differently. Unfortunately, our pretest data did not include such a ratio.

- Future research. Finally, we encourage future researchers to link self- and collective efficacy more strongly to the issue of private vs. public sphere behavior [3]. While it is quite plausible to assume that private sphere behavior (i.e., personal practice) may be less influenced by collective efficacy beliefs than public sphere behavior (i.e., civic action), some empirical evidence suggests otherwise. For example, Morton and colleagues [29] showed that collective efficacy predicted private sphere behavior (specifically, reducing household waste and non-green energy consumption); similarly, Lubell and colleagues [50], using a rational choice framework, came to similar conclusions (see also [30,31]). These findings show that collective efficacy is also important in private sphere settings-a view researchers have just begun to acknowledge (see also [11]). Yet, it would be informative and important to understand whether different aspects of efficacy relate to different private or public behaviors (see [51,52])—and whether such efficacy beliefs could be gauged by identification with groups that support sustainability causes.

\section{Conclusions}

To conclude, we believe that this study sheds a light on the boundary conditions of a potentially powerful route to sustainable intentions. Convincing individuals of their collective ability to achieve change could ultimately lead to collective efforts for attaining environmental goals, and it seems that one way to trigger these collective beliefs is through providing and developing moderately challenging goals.

Acknowledgments: We are thankful to Karen Hamann and Josephine Troeger for valuable comments on an earlier version of our manuscript. This research was partly funded by the Melton Foundation with a grant to the second author. 
Author Contributions: G.R. and E.A.J. conceived and designed the experiment; E.A.J. performed the experiments; G.R. and E.A.J. analyzed the data; G.R. wrote most parts of the paper.

Conflicts of Interest: The authors declare no conflict of interest.

\section{References}

1. Batalha, L.; Reynolds, K. ASPIRing to mitigate climate change: Superordinate identity in global climate negotiations. Polit. Psychol. 2012, 33, 743-760. [CrossRef]

2. Reese, G. Common human identity and the path to global climate justice. Clim. Chang. 2016, 134, 521-531. [CrossRef]

3. Stern, P. Toward a coherent theory of environmentally significant behavior. J. Soc. Issues 2000, 56, 407-424. [CrossRef]

4. Bamberg, S.; Möser, G. Twenty years after Hines, Hungerford, and Tomera: A new meta-analysis of psycho-social determinants of pro-environmental behavior. J. Environ. Psychol. 2007, 27, 14-25. [CrossRef]

5. Fielding, K.S.; McDonald, R.; Louis, W.R. Theory of planned behavior, identity and intentions to engage in environmental activism. J. Environ. Psychol. 2008, 28, 318-326. [CrossRef]

6. Schultz, P.W. The structure of environmental concern: Concern for self, other people, and the biosphere. J. Environ. Psychol. 2001, 21, 327-339. [CrossRef]

7. Van Zomeren, M.; Postmes, T.; Spears, R. Toward an integrative social identity model of collective action: A quantitative research synthesis of three socio-psychological perspectives. Psychol. Bull. 2008, 134, 504-535. [CrossRef] [PubMed]

8. Barth, M.; Jugert, P.; Fritsche, I. Still underdetected-Social norms and collective efficacy predict the acceptance of electric vehicles in Germany. Transp. Res. F 2016, 37, 64-77. [CrossRef]

9. Doran, R.; Hanss, D.; Larsen, S. Attitudes, efficacy beliefs, and willingness to pay for environmental protection when travelling. Tour. Hosp. Res. 2015, 15, 281-292. [CrossRef]

10. Doran, R.; Hanss, D.; Larsen, S. Intentions to make sustainable tourism choices: Do value orientations, time perspective, and efficacy beliefs explain individual differences? Scand. J. Hosp. Tour. 2016, 1-16. [CrossRef]

11. Cocking, C.; Drury, J. Generalization of Efficacy as a Function of Collective Action and Intergroup Relations: Involvement in an Anti-Roads Struggle. J. Appl. Soc. Psychol. 2004, 34, 417-444. [CrossRef]

12. Jugert, P.; Barth, M.; Greenaway, K.; Buechner, R.; Eisentraut, S.; Fritsche, I. Collective efficacy increases pro-environmental intentions through increasing personal efficacy. J. Environ. Psychol. 2016, 48, 12-23. [CrossRef]

13. Environment Protection and Heritage Council. Plastic Shopping Bags in Australia: National Plastic Bags Working Group Report to the National Packing Covenant Council; Environment Protection and Heritage Council: Adelaide, Australia, 2002. Available online: http://www.nepc.gov.au/system/files/resources/0c513e54-d968-ac04758b-3b7613af0d07/files/ps-pbag-rpt-npbwg-report-npcc-200212.pdf (accessed on 27 January 2017).

14. Steinmetz, Z.; Wollmann, C.; Schaefer, M.; Buchmann, C.; David, J.; Tröger, J.; Schaumann, G.E. Plastic mulching in agriculture. Trading short-term agronomic benefits for long-term soil degradation? Sci. Total Environ. 2016, 550, 690-705. [CrossRef] [PubMed]

15. Halden, R.U. Plastics and Health Risks. Annu. Rev. Public Health 2010, 31, 179-194. [CrossRef] [PubMed]

16. Thompson, R.; Moore, C.; vom Saal, F.; Swan, S. Plastics, the environment and human health: Current consensus and future trends. Philos. Trans. R. Soc. Lond. Ser. B 2009, 364, 2153-2166. [CrossRef] [PubMed]

17. Cherrier, H. Consumer identity and moral obligations in non-plastic bag consumption: A dialectical perspective. Int. J. Consum. Stud. 2006, 30, 515-523. [CrossRef]

18. Bandura, A. Self-efficacy mechanism in human agency. Am. Psychol. 1982, 37, 122-147. [CrossRef]

19. Bandura, A. Exercise of Human Agency through Collective Efficacy. Curr. Dir. Psychol. Sci. 2000, 9, 75-78. [CrossRef]

20. Ajzen, I. Perceived behavioral control, self-efficacy, locus of control, and the theory of planned behavior. J. Appl. Soc. Psychol. 2002, 32, 665-683. [CrossRef]

21. Bandura, A. Self-Efficacy: The Exercise of Control; Freeman: New York, NY, USA, 1997.

22. Tims, M.; Bakker, A.B.; Derks, D. Daily job crafting and the self-efficacy-Performance relationship. J. Manag. Psychol. 2014, 29, 490-507. [CrossRef] 
23. Riggio, H.R.; Weiser, D.A.; Valenzuela, A.M.; Lui, P.P.; Montes, R.; Heuer, J. Self-efficacy in romantic relationships: Prediction of relationship attitudes and outcomes. J. Soc. Psychol. 2013, 153, 629-650. [CrossRef] [PubMed]

24. LaForge-MacKenzie, K.; Sullivan, P.J.; Hansen, S.; Marini, M. The effect of attentional focus on the self-efficacy-performance relationship in a continuous running task: A pilot study. J. Exerc. Mov. Sport 2013, 45,1 .

25. Sitzmann, T.; Yeo, G. A Meta-Analytic Investigation of the Within-Person Self-Efficacy Domain: Is Self-Efficacy a Product of Past Performance or a Driver of Future Performance? Personal. Psychol. 2013, 66, 531-568. [CrossRef]

26. Lauren, N.; Fielding, K.S.; Smith, L.; Louis, W.R. You did, so you can and you will: Self-efficacy as a mediator of spillover from easy to more difficult pro-environmental behavior. J. Environ. Psychol. 2016, 48, 191-199. [CrossRef]

27. Fielding, K.S.; Hornsey, M.J. A social identity analysis of climate change and environmental attitudes and behaviors: Insights and opportunities. Front. Psychol. 2016, 7. [CrossRef] [PubMed]

28. Morton, T.A.; Rabinovich, A.; Marshall, D.; Bretschneider, P. The future that may (or may not) come: How framing changes responses to uncertainty in climate change communications. Glob. Environ. Chang. 2011, 21, 103-109. [CrossRef]

29. Homburg, A.; Stolberg, A. Explaining pro-environmental behavior with a cognitive theory of stress. J. Environ. Psychol. 2006, 26, 1-14. [CrossRef]

30. Chen, M.-F. Self-efficacy or collective efficacy within the cognitive theory of stress model: Which more effectively explains people's self-reported proenvironmental behavior. J. Environ. Psychol. 2015, 42, 66-75. [CrossRef]

31. Fritsche, I.; Jonas, E.; Ablasser, C.; Beyer, M.; Kuban, J.; Manger, A.-M.; Schultz, M. The power of we: Evidence for group-based control. J. Exp. Soc. Psychol. 2013, 49, 19-32. [CrossRef]

32. Greenaway, K.H.; Haslam, S.A.; Cruwys, T.; Branscombe, N.R.; Ysseldyk, R.; Heldreth, C. From "we" to "me": Group identification enhances perceived personal control with consequences for health and well-being. J. Personal. Soc. Psychol. 2015, 109, 53-74. [CrossRef] [PubMed]

33. Atkinson, J.W. Motivational determinants of risk-taking behavior. Psychol. Rev. 1957, 64, 359-372. [CrossRef] [PubMed]

34. Baron, R.A.; Mueller, B.A.; Wolfe, M.T. Self-efficacy and entrepreneurs' adoption of unattainable goals: The restraining effects of self-control. J. Bus. Ventur. 2016, 31, 55-71. [CrossRef]

35. Kanfer, R.; Ackerman, P.L. Aging, adult development, and work motivation. Acad. Manag. Rev. 2004, 29, 440-458.

36. Vancouver, J.B.; More, K.M.; Yoder, R.J. Self-efficacy and resource allocation: Support for a nonmonotonic, discontinuous model. J. Appl. Psychol. 2008, 93, 35. [CrossRef] [PubMed]

37. Van Zomeren, M.; Spears, R.; Fischer, A.H.; Leach, C.W. Put your money where your mouth is! Explaining collective action tendencies through group-based anger and group efficacy. J. Personal. Soc. Psychol. 2004, 87, 649-664. [CrossRef] [PubMed]

38. Bator, R.J.; Tabanico, J.J.; Walton, M.L.; Schultz, P.W. Promoting energy conservation with implied norms and explicit messages. Soc. Influ. 2014, 9, 69-82. [CrossRef]

39. Goldstein, N.J.; Cialdini, R.B.; Griskevicius, V. A room with a viewpoint: Using social norms to motivate environmental conservation in hotels. J. Consum. Res. 2008, 35, 472-482. [CrossRef]

40. Hamann, K.R.; Reese, G.; Seewald, D.; Loeschinger, D.C. Affixing the theory of normative conduct (to your mailbox): Injunctive and descriptive norms as predictors of anti-ads sticker use. J. Environ. Psychol. 2015, 44, 1-9. [CrossRef]

41. Nolan, J.M.; Schultz, P.W.; Cialdini, R.B.; Goldstein, N.J.; Griskevicius, V. Normative social influence is underdetected. Personal. Soc. Psychol. Bull. 2008, 34, 913-923. [CrossRef] [PubMed]

42. Reese, G.; Loew, K.; Steffgen, G. A towel less: Social norms enhance pro-environmental behavior in hotels. J. Soc. Psychol. 2014, 154, 97-100. [CrossRef] [PubMed]

43. Hanss, D.; Böhm, G.; Doran, R.; Homburg, A. Sustainable consumption of groceries: The importance of believing that one can contribute to sustainable development. Sustain. Dev. 2016, 24, 357-370. [CrossRef] 
44. Mancha, R.; Muniz, K.; Yoder, C. Studying Executives' Green Behaviors: An Environmental Theory of Planned Behavior. Unpublished Manuscript. 2014. Available online: http://aisel.aisnet.org/cgi/ viewcontent.cgi?article=1574\&context=amcis2014 (accessed on 27 January 2017).

45. Han, H.; Hsu, L.; Sheu, C. Application of the Theory of Planned Behavior to green hotel choice: Testing the effect of environmental friendly activities. Tour. Manag. 2010, 31, 325-334. [CrossRef]

46. Hayes, A.F. Introduction to Mediation, Moderation, and Conditional Process Analysis; Guildford Press: New York, NY, USA, 2013.

47. Truelove, H.B.; Carrico, A.R.; Weber, E.U.; Raimi, K.T.; Vandenbergh, M.P. Positive and negative spillover of pro-environmental behavior: an integrative review and theoretical framework. Glob. Environ. Chang. 2014, 29, 127-138. [CrossRef]

48. Sikor, T.; Martin, A.; Fisher, J.; He, J. Toward an empirical analysis of justice in ecosystem governance. Conserv. Lett. 2014, 7, 524-532. [CrossRef]

49. Reese, G.; Jacob, L. Principles of environmental justice and pro-environmental action: A two-step process model of moral anger and responsibility to act. Environ. Sci. Policy 2015, 51, 88-94. [CrossRef]

50. Lubell, M.; Zahran, S.; Vedlitz, A. Collective action and citizen responses to global warming. Polit. Behav. 2007, 29, 391-413. [CrossRef]

51. Alisat, S.; Riemer, M. The environmental action scale: Development and psychometric evaluation. J. Environ. Psychol. 2015, 43, 13-23. [CrossRef]

52. Dono, J.; Webb, J.; Richardson, B. The relationship between environmental activism, pro-environmental behavior and social identity. J. Environ. Psychol. 2010, 30, 178-186. [CrossRef]

(C) 2017 by the authors; licensee MDPI, Basel, Switzerland. This article is an open access article distributed under the terms and conditions of the Creative Commons Attribution (CC BY) license (http:/ / creativecommons.org/licenses/by/4.0/). 